\title{
An Empirical Study of the Role of Magnetic, Geometric, and Tissue Properties on the Turning Radius of Magnetically Driven Screws
}

\author{
Nathan D. Nelson, Jessica Delacenserie, and Jake J. Abbott
}

\begin{abstract}
Previous work in the open-loop behavior of magnetically driven screws in soft tissue has focused on the impact of magnetic field rotation speed and pitch angle, measured between the field's rotation axis and the screw's principle axis, on the average magnetic torque, both in-plane and out-of-plane. However, prior work did not rigorously consider the role of screw geometry and tissue material properties on the resulting trajectory. This study seeks to develop a plausible empirical model from experimental measurements in an agar gel tissue phantom for use in further research and feasibility studies of magnetic screws for in vivo applications. Non-dimensional parameters representing rotation speed, pitch angle, screw size, magnetic strength, and tissue stiffness are varied orthogonally. Circular trajectories are fitted to the resulting recorded trajectories. Using physical insight and observation of the trends of the non-dimensional turning radius/curvature, simple models for each parameter group are proposed. A final empirical model is then proposed to unify the individual models.
\end{abstract}

\section{INTRODUCTION}

There is significant interest in the use of microscale and mesoscale robotic devices in minimally invasive medicine [1]. A great deal of research has explored the use of magnetics as a wireless means of control and power transfer. Much of magnetically driven small-scale robotics has focused on swimming locomotion within a low-Reynolds-number fluid environment [2]. However, a large amount of the human body is inaccessible to swimming robots. It is desirable to access areas that contain soft tissues, such as the liver, the brain, the prostate, and the eye. A permanent-magnet screw or a nonmagnetic screw with an affixed permanent magnet, with the magnet's dipole moment orthogonal to the screw's rotation axis, can be driven through soft tissue using a rotating magnetic field. In order to develop reliable motion planning methods, the locomotion of the screw within the tissue needs to be well understood.

Researchers at Tohoku University have explored screwtype machines in fluids and soft tissues in vitro in various media, including animal tissue. "Magnetic machines" with spiral body-wrapped features were fabricated and controlled using rotating magnetic fields in agar gel and bovine meat [3], [4]. These experiments were limited to straight runs but showed promise for use in soft-tissue environments. Possible applications of the spiral-type machines were explored, including utilizing high-frequency magnetic fields to induce hyperthermia in an attached ferrite body, as well as

This work is supported by the National Science Foundation under Grant Nos. 0952718 and 0654414

The authors are with the Department of Mechanical Engineering, University of Utah, UT 84112, USA. \{nathan.d.nelson, jake.abbott\}@utah.edu

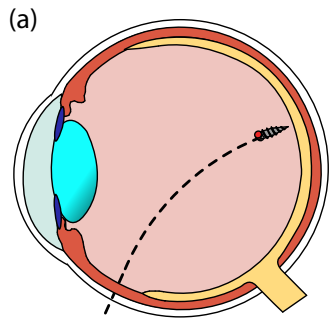

(b)

Fig. 1: Magnetically driven screws operating in soft-tissue environments could deploy a variety of localized therapies. (a) A screw capable of traversing the vitreous could deliver clot-busting enzymes to the retina without necessitating a vitrectomy. (b) A screw could drive into the center of a tumor within an organ to deliver hyperthermia or brachytherapy without causing large-scale tissue deformation.

to transport payload carriers [5]-[7]. The results suggest that straight-run performance of the machines should not be significantly hindered by attaching required hardware for specific targeted therapies. The control of multiple spiraltype machines was studied with bodies that exhibit different frictional rotational characteristics [6], [8]. This enabled the individual control of several machines by varying the rotational field frequencies about the machines' individual step-out frequencies (i.e., the frequencies above which the screw cannot remain synchronized with the rotating field) Further refinement of machine design included the addition of cutting blades at the body tip for traveling in the liver [9][11]. Although some of the studies involved making turns within agar gel, the problem of executing turns in soft tissue was formally addressed in [8]. From the theoretical torque equations and predicted body rolling, an empirical model was proposed that predicted the average torques on the robot. The average turning torque was shown to increase to a maximum when the field's axis of rotation was pitched to $60^{\circ}$ from the screw's body axis, regardless of screw size parameters. An empirical relation between average turning radius and pitch angle showed a minimum turning radius at $60^{\circ}$ pitch angle, corresponding to the maximum torque as expected.

Our group has recently extended the exploration of [8] by studying the effects of pitch angle and speed on the average turning torque predicted in the model equations [12]. We found that desired in-plane torque required for a desired turning maneuver is always coupled with a nonideal out-of-plane torque. We found that certain combinations of speed and pitch angle resulted in the non-ideal torque dominating, resulting in an unstable screw trajectory, and that 
certain ranges of speed and pitch angle exist that mitigate this effect. However, that work explored what is a purely magnetic phenomenon; it did not consider the effect of device geometry nor specific tissue parameters on turning radius in the event of a stable turn.

Prior experimental results have been somewhat ad hoc, and to date it is not possible to answer the question: "For a given magnetic screw moving through a given soft tissue, using a given rotating magnetic field, what will the turning radius of the screw be?" This paper provides an empirical study that aims to answer this question. Creating such a model, either empirically or theoretically (although the latter is challenging, considering the complexity of tissue mechanics), is the first step toward answering two more interesting and constructive questions: "For a given magnetic screw moving through a given soft tissue, what rotating magnetic field should be generated to cause a desired turning radius of the screw?" and "What is the optimal set of magneticscrew parameters for use in a given application in a given soft-tissue environment?" Our long-term goal is to pursue the answers to these questions.

In this paper, we begin by conducting dimensional analysis using the Buckingham Pi theorem [13] to determine a set of dimensionless parameters that fully characterize magneticscrew turning. Using dimensionless variables enables us to understand how experimental results obtained by us and by others generalize to a wide range of magnetic screws and soft tissues not explicitly tested. The dimensional analysis is followed by a set of experiments to measure the turning radius (and inversely, the turning curvature) of a set of magnetic screws moving through agar under a variety of magnetic-field scenarios. Analysis of this data enables us to create a simple model that predicts the role of the various experimental parameters on the turning radius. The results can be used to inform future studies to characterize the phenomenon more completely.

The dimensional analysis is presented in Section II. The experimental procedure is given in Section III, with physical set-up described in Section III-A, and the method and results discussed in Section III-B. The empirical model is created in Section IV.

\section{DIMENSIONAL ANALYSIS}

We wish to consider the case of a screw with length $L$ $\{\mathrm{m}\}$ and diameter $D\{\mathrm{~m}\}$ being driven in tissue with an elastic modulus $E\left\{\mathrm{~Pa}=\mathrm{kg} \cdot \mathrm{m}^{-1} \cdot \mathrm{s}^{-2}\right\}$. There is affixed a permanent magnet with a dipole moment of magnitude $|\mathbf{m}|$ $\left\{\mathrm{A} \cdot \mathrm{m}^{2}=\mathrm{C} \cdot \mathrm{s}^{-1} \cdot \mathrm{m}^{2}\right\}$. It is being driven with a magnetic field with magnitude $|\mathbf{B}|\left\{\mathrm{T}=\mathrm{kg} \cdot \mathrm{C}^{-1} \mathrm{~s}^{-1}\right\}$ rotating at a constant rate $\Omega\left\{\mathrm{rad} \cdot \mathrm{s}^{-1}\right\}$ below a limiting step-out frequency $\omega_{s o}$ $\left\{\mathrm{rad} \cdot \mathrm{s}^{-1}\right\}$ and being steered by pitching its axis of rotation at a constant angle $\psi\{\operatorname{rad}\}$ from the screw's body axis. We want to characterize the turning radius $r\{\mathrm{~m}\}$ made by the screw trajectory in the tissue.

Using dimensional analysis, the dimensionless turning radius $\mathcal{R}$, and inversely, the dimensionless curvature $\mathcal{C}$,

$$
\mathcal{R}=\frac{r}{D} \leftrightarrow \mathcal{C}=\frac{D}{r}
$$

is a function of four dimensionless parameters. The first is a dimensionless rotation rate term:

$$
\mathcal{F}=\frac{\Omega}{\omega_{\text {so }}},
$$

which encodes the ratio of body rotation speed to the stepout frequency. The second parameter encodes the ratio of magnetic torque to tissue stiffness:

$$
\mathcal{M}=\frac{|\mathbf{m}||\mathbf{B}|}{D^{3} E} .
$$

The third is the screw's length-to-diameter ratio:

$$
\mathcal{L}=\frac{L}{D},
$$

which encodes the screw geometry. Finally, the last parameter is the steering angle $\psi$, which is already dimensionless. It should be noted that the four dimensionless independent groups chosen above are not unique, and other equally valid sets of four could be chosen. The groupings were chosen to encode some physically meaningful quantities (as perceived by the authors).

The Buckingham Pi theorem [13] states that for a given phenomenon or system, the dimensionless parameter groups can be expressed as functions of the other independent groups. Thus the dimensionless curvature can be thought of as

$$
\mathcal{C}=f(\mathcal{F}, \mathcal{M}, \mathcal{L}, \psi)
$$

The function in (5) is at most a function in four variables. The Buckingham Pi theorem does not tell us anything about the form of the function, or whether every independent group is important. For this, we turn to experiments.

\section{EXPERIMENTS}

\section{A. Experimental Setup}

The control of the magnetic fields is done using a set of three nested Helmholtz-coil pairs orthogonally arranged so that each pair commands a basis vector of the total magnetic field (Fig. 2a). The coils are wrapped around an acrylic frame with 14 AWG insulated copper magnet wire. The distance separating each pair is equal to the coil radius. Although the details of construction of the coils are given in [14], for completeness the parameters are listed in Table I. The field in the center of the coils is approximately uniform. Each coil pair is controlled using an Advanced Motion Controls S16A8 PWM amplifier and powered by an Advanced Motion Controls PS2x300W power supply. The amplifiers are set to act as current sources given a commanded voltage via a Sensoray s626 DAQ card connected to a desktop PC. Video of the coil workspace is captured using a Basler A602FC camera outfitted with a Computar MLH-10X wide-angle lens.

The screws are altered brass screws that have the heads removed (Fig. 2b). Cubic NdFeB N50-grade permanent magnets of $1 \mathrm{~mm}$ on a side with estimated magnet dipole of $0.0011 \mathrm{~A} \cdot \mathrm{m}^{2}$ are epoxied onto the smoothed cut surface with the dipole orthogonal to the screw's body axis. The 


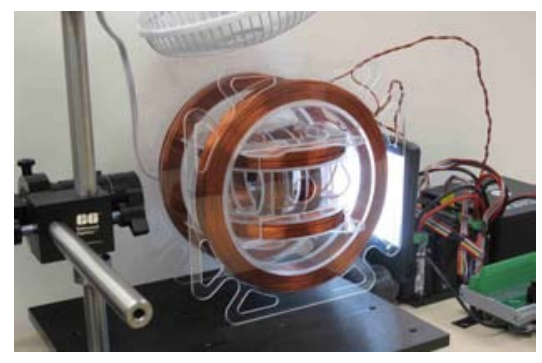

(a)

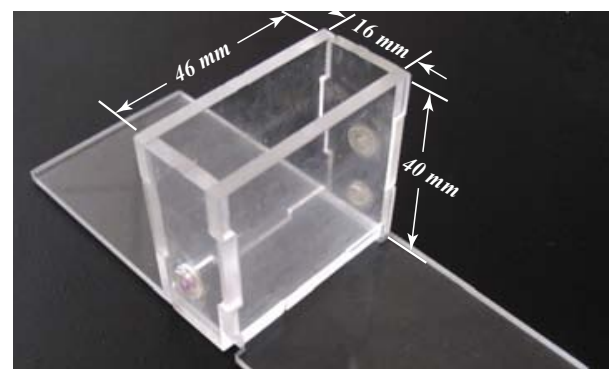

(c)

Fig. 2: (a) The nested Helmholtz coils and screws used to conduct experiments. See Table I for parameters. (b) The four screws used in experiments are made of brass with an affixed $1 \mathrm{~mm}$ cubic permanent magnet. See Table II for parameters. (c) Blocks of agar gel are held in a tank made of acrylic and designed with interchangeable pieces that allow for features to be added such as screw ports. The tank dimensions maximize the workspace of the screw runs within the center of the Helmholtz coils.

screw dimensions are given in Table II. Measurements of the length of the screw include the attached magnet, since then entire rigid body must rotate within the tissue as the screw turns.

Agar gel is used as a tissue phantom to allow for computer vision while mimicking various soft tissues such as ocular vitreous humor [15], [16], brain tissue [17], and breast tissue [18]. Agar was previously used for magnetic-screw studies in [3], [9], [12]. The agar gel is made in two stages: a boiling stage at approximately double strength, and a dilution stage to final concentration. The two-stage method was used to reduce heat load on the refrigeration system and ensure tighter control on the final concentration. The agar is chilled in a household refrigerator for 12 hours until set. The agar gel is then cut into $45 \mathrm{~mm} \times 30 \mathrm{~mm} \times 15 \mathrm{~mm}$ blocks.

To determine the properties of the prepared agar gel, compression testing was performed across 16 identically prepared $20 \mathrm{~mm}$ diameter cylindrical samples on an Instron 5943 mechanical testing system. Each sample was compressed to $5.0 \%$ strain with a rate of $2.0 \mathrm{~mm} / \mathrm{min}$. This strain and strain rate were selected based on estimates of the agar strain when the screw is traveling through at a moderate rate. The mean elastic modulus was determined to be $2.35 \pm 0.65 \mathrm{kPa}$ for the prepared agar samples.
TABLE I: Parameters of Helmholtz Coils

\begin{tabular}{|c|c|c|c|c|c|c|}
\hline $\begin{array}{c}\text { Coil } \\
\text { Set }\end{array}$ & $\begin{array}{c}\text { Radius } \\
(\mathrm{mm})\end{array}$ & $\begin{array}{c}\text { Width } \\
\text { Wraps }\end{array}$ & $\begin{array}{c}\text { Depth } \\
\text { Wraps }\end{array}$ & $\begin{array}{c}\text { Total } \\
\text { Wraps }\end{array}$ & $\begin{array}{c}\text { Resist. } \\
(\Omega)\end{array}$ & $\begin{array}{c}\text { Induct. } \\
(\mathrm{mH})\end{array}$ \\
\hline \hline Inner & 44 & 9 & 7 & 63 & 0.5 & 0.944 \\
\hline Middle & 69 & 9 & 11 & 99 & 0.9 & 3.78 \\
\hline Outer & 98 & 13 & 11 & 143 & 1.6 & 12.2 \\
\hline
\end{tabular}

TABLE II: Screw geometric parameters.

\begin{tabular}{|c|c|c|c|}
\hline $\begin{array}{c}\text { Screw } \\
\text { No. }\end{array}$ & $\begin{array}{c}\text { Length } \\
(\mathrm{mm})\end{array}$ & $\begin{array}{c}\text { Pitch } \\
(\mathrm{mm})\end{array}$ & $\begin{array}{c}\text { Diameter } \\
(\mathrm{mm})\end{array}$ \\
\hline \hline 1 & 4.0 & 0.87 & 1.4 \\
\hline 2 & 4.6 & 0.87 & 1.4 \\
\hline 3 & 5.7 & 0.87 & 1.4 \\
\hline 4 & 6.6 & 0.87 & 1.4 \\
\hline
\end{tabular}

For each run, a block of agar is placed within an acrylic holding tank (Fig. 2c) fitted with interchangeable sides with screw ports made using small sections of soft plastic tubing epoxied into a through-hole drilled to ensure the screw enters the agar block approximately parallel with horizontal.

\section{B. Methods and Results}

1) Methodology: A typical run begins by observing the step-out frequency within the agar, then selecting a magnetic field rotation rate at the required fraction of step out. A human operator observes a real-time video image of the experiment. As the screw moves along its trajectory, the operator manually adjusts overlayed parallel lines in the video to be parallel to the rotation axis of the screw. This information about the axis is given to the magnetic-fieldgeneration system so that it can maintain the pitch of the rotating magnetic field at a constant value with respect to the screw. Once the screw has reached a surface of the agar block, the field rotation is stopped by the operator.

The resulting videos are processed using a blob detection algorithm that identifies the screw using pixel area and aspect ratio as identifiers and outputs the location of the center of the bounding rectangle and angle of rotation in the camera plane. Extraneous blobs are removed and the data transformed into the world-frame units. Finally, the points of the trajectory are fit to a least-squares-error in-plane circle using the Curve Fitting Toolbox in MATLAB (Fig. 3).

The dimensionless groups were varied orthogonally to explore the turning radius/curvature behavior. This was done by varying the nominal parameters $(\mathcal{L}=4.1, \psi=0.393, \mathcal{M}$ $=1.33, \mathcal{F}=0.30$ ) one at a time while holding the other three parameters constant. The resulting data points are plotted in Fig. 4 and discussed in Section IV.

2) Sources of Experimental Error: The significant variability (or noise) in driving and turning screws in tissue has been observed previously in [3], [4], and is also seen in the results from this study. This suggests that the agar gel microstructure may introduce irregularities in the screw 


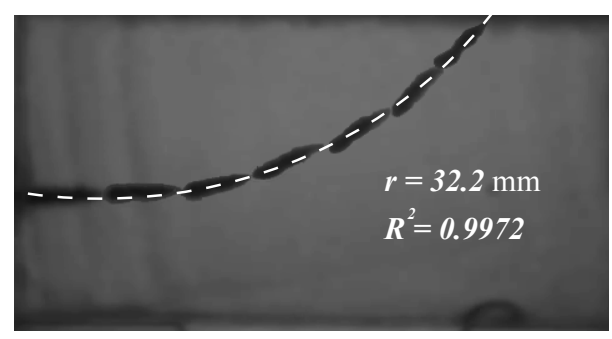

Fig. 3: The trajectory of Screw No. 3 run at $\mathcal{F}=0.25$ (i.e., $25 \%$ of step-out frequency), $\psi=0.175 \mathrm{rad}$ (i.e., $10^{\circ}$ ), and $|\mathbf{B}|=9.8 \mathrm{mT}$, in $0.5 \mathrm{wt} . \%$ agar gel.

trajectory. Additionally, the agar gel parameters may vary with temperature; efforts were made to minimize this effect by keeping unused blocks refrigerated until ready for use. Gravity acting on the gel can create a hydrostatic pressure differential in the vertical direction of the blocks, and the resulting compression could result in changes in parameters across the block. Since the vertical direction is the direction of turning, this could result in undesired noise, but experimental runs were conducted quickly after placing the blocks vertically to minimize this source of error. If the agar and screw interaction is not entirely linearly elastic (i.e., the static torque response of the screw in agar gel exhibits viscoelastic creep or is nonlinear in nature), the dimensionless parameters would not encapsulate this behavior. Finally, it is possible that the trajectories are sensitive to the initial rotation of the screw about its axis, relative to the turning axis. All of the above effects could contribute to the large amount of variance within the experimental data.

\section{EMPIRICAL MODEL}

\section{A. Dimensionless Rotation Speed Parameter $\mathcal{F}$}

Screw No. $3(\mathcal{L}=4.1)$ was run with a pitch angle of $20^{\circ}$ $(\psi=0.35)$ and a magnetic field magnitude $7.8 \mathrm{mT}(\mathcal{M}=$ 1.33). The rotation speeds were varied from $10 \%$ to $90 \%$ of measured step-out frequency. The plot of the data is shown in Fig. 4a.

In [12], it was observed that increasing field rotation speed resulted in less time-averaged magnetic torque for turning. Similarly, it was shown that the slower the rotation rate, the higher the average turning torque. This implies that the $\mathcal{R}$ should increase to a maximum as $\mathcal{F}$ increases to 1 (screw step out) and decrease towards a non-zero minimum as $\mathcal{F}$ decreases to zero (representing quasistatic turning). Based on the observed trend in Fig. 4a, a simple model that fulfills these requirements and fits the data is a straight affine line:

$$
\mathcal{R}=K_{F 1} \mathcal{F}+K_{F 2},
$$

where $K_{F 1}$ and $K_{F 2}$ are constants.

\section{B. Dimensionless Pitch Angle Parameter $\psi$}

Screw No. $3(\mathcal{L}=4.1)$ was run at a rotation rate of $30 \%$ of measured step-out frequency $(\mathcal{F}=0.30)$ and a magnetic field magnitude of $7.8 \mathrm{mT}(\mathcal{M}=1.33)$. The pitch angles were varied from $0^{\circ}$ to $70^{\circ}$. The data plot is shown in Fig. $4 \mathrm{~b}$.
In [8] and [12], the maximum average magnetic turning torque occurs at a pitch angle of $60^{\circ}$. In [12], undesirable outof-plane turning torque increases as pitch angle increases. Thus there is an upper limit to the effective modeling of inplane curvature measurements. This is clearly indicated in Fig. $4 \mathrm{~b}$ by the increase in variance above approximately $30^{\circ}$. Additionally, if the pitch angle is zero, there should be no turning and a zero curvature. Finally, since the pitch angle is a periodic variable, it makes sense to select a trigonometric function to fit the data. Thus, a simple model that fulfills these requirements is a sinusoid, specifically one that peaks at a value of $\psi=60^{\circ}$ :

$$
\mathcal{C}=K_{\psi} \sin \left(\frac{3 \psi}{2}\right)
$$

where $K_{\psi}$ is a constant.

\section{Dimensionless Magnetic Parameter $\mathcal{M}$}

Screw No. $3(\mathcal{L}=4.1)$ was run at a speed of $30 \%$ of measured step-out frequency $(\mathcal{F}=0.30)$ with a pitch angle of $20^{\circ}(\psi=0.35)$. The magnetic field magnitude is varied with values $\{4.9,5.8,6.8,7.8,8.7,9.8\} \mathrm{mT}$. The plot of data is shown in Fig. 4c.

Considering the definition of $\mathcal{M}$ in (3), if $\mathcal{M}$ were increased to an arbitrarily large amount, then the magnetic torque would increase toward infinity, relative to the tissue stiffness. As long as there is no limit to the amount of torque applied, it stands to reason that no amount of tissue stiffness will stop the screw's motion from nearly instantaneously tracking the torque input. Thus curvature would be expected to go to infinity. Additionally, if the tissue stiffness were to increase such that $\mathcal{M}$ goes to zero, the curvature of the resulting path should go to zero as well. Despite the large amount of variance in each level, the experimental data in Fig. $4 \mathrm{c}$ seems to exhibit a linear trend. Thus, a simple model would be a straight line through the origin:

$$
\mathcal{C}=K_{M} \mathcal{M},
$$

where $K_{M}$ is a constant.

\section{Dimensionless Screw Length Parameter $\mathcal{L}$}

The screws shown in Fig. $2 b$ with parameters given in Table II were each run at a rotation speed of $30 \%$ of measured step-out frequency $(\mathcal{F}=0.30)$, a magnetic field magitude of $7.8 \mathrm{mT}(\mathcal{M}=1.33)$, and steered with a pitch angle of $20^{\circ}(\psi=0.35)$. The data plot is shown in Fig. 4d.

If the screw is treated as an equivalent cylinder embedded in material, then as the length increases more material is distributed along the screw body to resist rotation. Thus, as $\mathcal{L}$ increases toward infinity, $\mathcal{R}$ would also be expected to approach infinity. Similarly, if the length were to decrease or the diameter increase without bound, then more material in the radial direction would counter rotation relative to the size of the screw. In this sense, a value of $\mathcal{L}=1$ can be thought of as a null aspect ratio. In practice a value of $\mathcal{L}<1$ will not be realizable. The plot of data in Fig. $4 d$ shows a trend that 


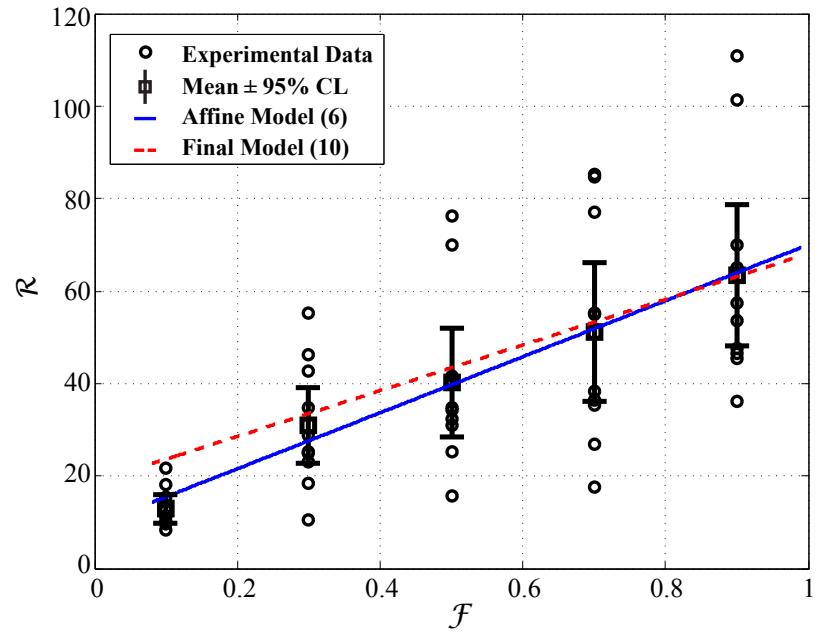

(a)

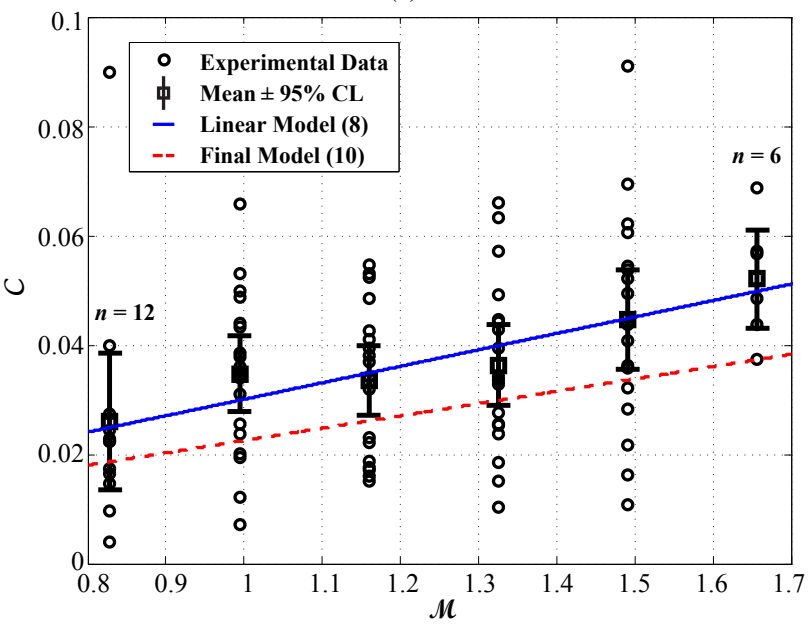

(c)

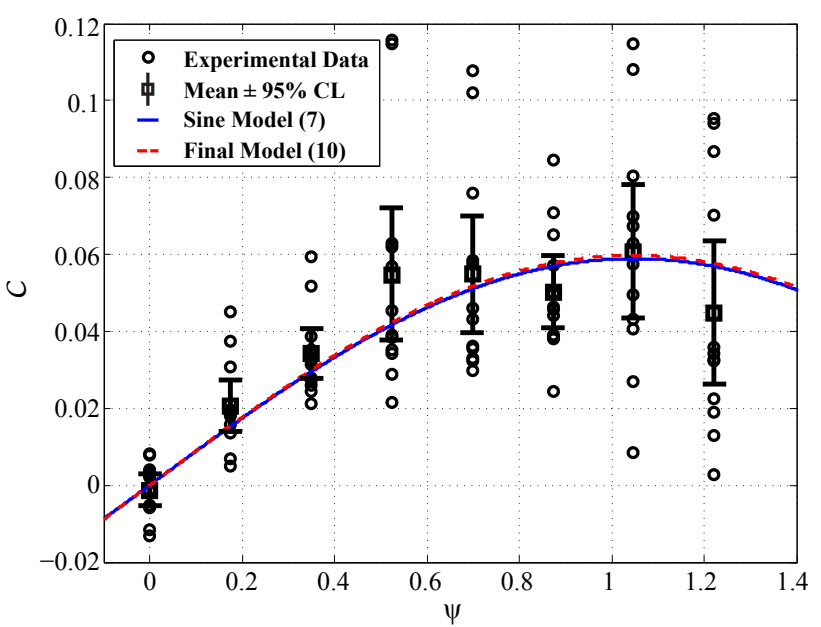

(b)

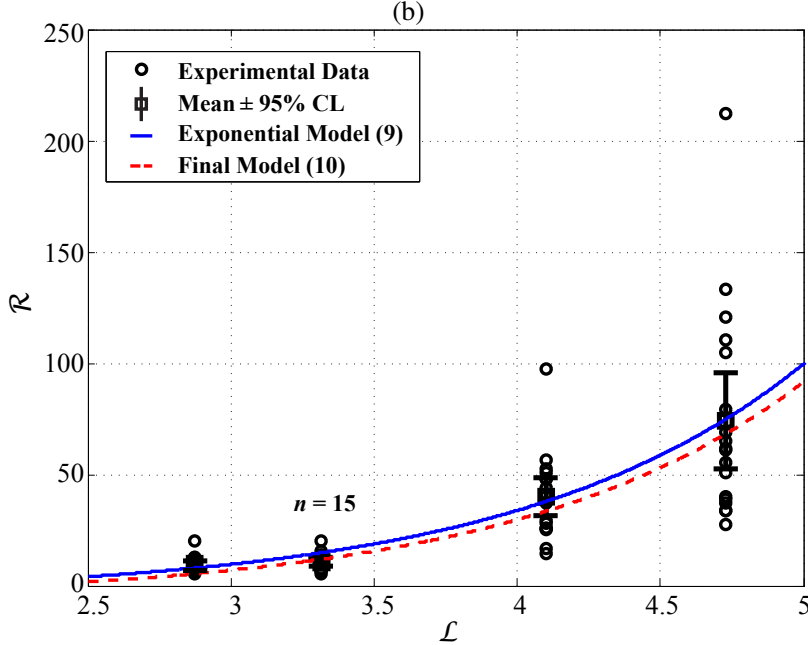

(d)

Fig. 4: (a) Experimental data for $\mathcal{R}$ as $\mathcal{F}$ is varied $(\mathcal{L}=4.1, \psi=0.35, \mathcal{M}=1.33, n=10$ points per level). The linear trend is shown with $R^{2}$ of 0.9871 . (b) Experimental data for $\mathcal{C}$ as $\psi$ is varied $(\mathcal{L}=4.1, \mathcal{F}=0.30, \mathcal{M}=1.33, n=12$ points per level). The sinusoidal trend is shown with $R^{2}$ of 0.8623. (c) Experimental data for $\mathcal{C}$ as $\mathcal{M}$ is varied $(\mathcal{L}=4.1, \mathcal{F}=0.30$, $\psi=0.35, n=18$ points per level, except where otherwise noted). The affine linear trend is shown with $R^{2}$ of 0.9804 . (d) Experimental data for $\mathcal{R}$ as $\mathcal{L}$ is varied $(\mathcal{M}=1.33, \mathcal{F}=0.30, \psi=0.35, n=18$ points per level, except where noted). The exponential trend is shown with $R^{2}$ of 0.9907 . For each plot, the error bars represent the $95 \%$ confidence limit on the mean.

appears to be less sensitive in the lower $\mathcal{L}$ values. A simple model that describes the observed trend is an exponential function:

$$
\mathcal{R}=K_{L 1} e^{\mathcal{L}-1}+K_{L 2},
$$

where $K_{L 1}$ and $K_{L 2}$ are constants.

\section{E. Final Empirical Model}

Now that partial models have been identified, we propose an empirical model that captures the essence of each of the four partial models:

$$
\mathcal{C}=\frac{\sigma_{1} \mathcal{M} \sin \left(\frac{3 \psi}{2}\right)}{\left(\mathcal{F} e^{\mathcal{L}-1}+\sigma_{2} \mathcal{F}+\sigma_{3} e^{\mathcal{L}-1}+\sigma_{4}\right)},
$$

where $\sigma_{1}, \sigma_{2}, \sigma_{3}$, and $\sigma_{4}$ are constants. The model is given in terms of curvature $\mathcal{C}$ because a zero curvature (i.e., a straight path) is realizable in practice, whereas a zero turning radius $\mathcal{R}$ (i.e., an infinitely tight circle) is not.

The constants $K_{i}$ in (6)-(9) were fitting parameters for the specific nondimensional data sets; they are implied functions of the dimensionless groups, and would be different for other combinations of values. The constants $\sigma_{i}$ of the final model are intended to be universal constants that should be applicable to a wider range of parameters not explicitly tested in our experiment. The mix of $\mathcal{L}$ and $\mathcal{F}$ in the denominator account for interaction effects that could be present, given the simple models in (6) and (9). For instance, it is expected that at constant $\mathcal{F}$, the influence of $\mathcal{F}$ on $\mathcal{L}$ would be contained in the constants $K_{F 1}$ and $K_{F 2}$ of (6). 
To find the dimensionless constants for the final empirical model, we used the MATLAB function fminsearch, which uses the Nelder-Mead simplex search algorithm. The residuals sum-of-squares error was minimized between the matched data trends in each set and the final empirical model for the same values. The constants were found to be $\sigma_{1}=0.478, \sigma_{2}=-6.56, \sigma_{3}=0.266, \sigma_{4}=73.7$. The model predictions are plotted alongside the data trends in Figs. $4 \mathrm{a}-4 \mathrm{~d}$. It can be seen that the final model captures the trends observed in all four experiments, even though there is some observed difference.

The empirical model in its presented form can be used to conduct basic feasibility studies and make better informed design decisions about the realistic capabilities of magnetic screws in soft tissue. For example, if the magnetic field magnitude were to be doubled, the maximum curvature would also double. Similarly, if the tissue were twice as stiff, the maximum curvature would be halved. Furthermore, if the screw length were to be halved, the maximum curvature would increase by more than a factor of two.

This empirical model has been derived by fitting trends to experimental data conducted in agar gel as a tissue phantom. Thus it cannot be said that this model would work for any soft tissue, but rather, soft tissues that exhibit similar characteristics to agar gel. The model is proposed as a plausible relationship among the nondimensional parameters within the studied region of values. Further refinement of experimental procedures, which include other tissues and tissue phantoms, and development of numerical finite-element simulations, will ultimately lend more confidence to the empirical model. Additional experimental data should be taken to validate the final model and provide estimates with higher confidence for the model parameters.

\section{CONCLUSIONS}

Previous work in studying the trajectories of magnetically driven screws in soft tissue has focused on the role that magnetic field rotation rate and pitch angle plays on the average magnetic torque and turning radius. This paper sought to find an empirical model from experimental data collected in agar gel to gain insight on how screw geometry and tissue properties, in addition to the previously considered effects, affect turning radius. Dimensionless groups were formed that represent rotation speed, pitch angle, screw size, magnetic strength, and tissue stiffness. These groups were varied orthogonally at different levels, and simple models were suggested based on observed trends of the experimental turning radii combined with physical insight about the expected behavior of the screws in tissue. The final model is believed to be a plausible representation that will aid in further experimental studies, physical modeling, and numerical simulation to ultimately result in an improved model with a high degree of confidence for magnetic-screw path planning in soft tissue.

\section{ACKNOWLEDGMENT}

The authors would like to thank Jeremy Greer and Dr. Eberhard Bamberg for assistance in fabrication of the mag- netic screws, Alex Williams and Dr. Brittany Coats for experimental determination of the properties of the agar gel used in the experiments, Andrew Petruska for assistance in estimation of the magnet dipole moment of the screw magnets, and Arthur Mahoney for fruitful discussions about the physics of the magnetic actuation of screws.

\section{REFERENCES}

[1] B. J. Nelson, I. K. Kaliakatsos, and J. J. Abbott, "Microrobots for minimally invasive medicine," Annual Review of Biomedical Engineering, vol. 12, pp. 55-85, 2010 .

[2] J. J. Abbott, K. E. Peyer, M. Cosentino Lagomarsino, L. Zhang, L. X. Dong, I. K. Kaliakatsos, and B. J. Nelson, "How should microrobots swim," Int. J. Robotics Research, vol. 28(11-12), pp. 1434-1447, 2009.

[3] K. Ishiyama, M. Sendoh, A. Yamazaki, and K. I. Arai, "Swimming micro-machine driven by magnetic torque," Sensors and Actuators A, vol. 91, no. 1-2, pp. 141-144, 2001

[4] K. Ishiyama, K. I. Arai, M. Sendoh, and A. Yamazaki, "Spiral-type micro-machine for medical applications." Journal of Micromechatronics, vol. 2, no. 1, pp. $77-86,2003$.

[5] M. Sendoh, K. Ishiyama, K. Arai, M. Jojo, F. Sato, and H. Matsuki, "Fabrication of magnetic micromachine for local hyperthermia," IEEE Transactions on Magnetics, vol. 38, no. 5, pp. 3359 - 3361, 2002.

[6] K. Ishiyama, M. Sendoh, and K. I. Arai, "Magnetic micromachines for medical applications," Journal of Magnetism and Magnetic Materials, vol. 242-245, pp. $41-46,2002$.

[7] F. Sato, M. Jojo, H. Matsuki, T. Sato, M. Sendoh, K. Ishiyama, and K. Arai, "The operation of a magnetic micromachine for hyperthermia and its exothermic characteristic," IEEE Transactions on Magnetics, vol. 38 , no. 5 , pp. $3362-3364,2002$.

[8] M. Sendoh, K. Ishiyama, and K. I. Arai, "Direction and individual control of magnetic micromachine," IEEE Transactions on Magnetics, vol. 38, no. 5, pp. 3356-3358, 2002

[9] M. Sendoh, A. Yamazaki, A. Chiba, M. Soma, K. Ishiyama, and K. I. Arai, "Spiral type magnetic micro actuators for medical applications," in Proceedings of the 2004 International Symposium on MicroNanoMechatronics and Human Science, 2004, pp. 319 - 324.

[10] M. Soma, M. Sendoh, K. Ishiyama, and K. I. Arai, "Fabrication of a magnetic micromachine capable of moving in a liver," Journal of the Magnetics Society of Japan, vol. 28, pp. 441-444, 2004.

[11] M. Soma, M. Sendoh, K. Ishiyama, K. Shoji, H. Watanabe, and K. I. Arai, "Direction control of a magnetic micromachine capable of moving in a liver," Journal of the Magnetics Society of Japan, vol. 29, pp. 594-597, 2005.

[12] A. W. Mahoney, N. D. Nelson, E. M. Parsons, and J. J. Abbott, "Nonideal behaviors of magnetically driven screws in soft tissue," in Proc. of IEEE/RSJ Int. Conf. Intelligent Robots and Systems, 2012, pp. 35593564

[13] K. Hutter, Continuum methods of physical modelling: mechanics of continua, dimensional analysis, turbulance. Springer, 2004.

[14] A. W. Mahoney, J. C. Sarrazin, E. Bamberg, and J. J. Abbott, "Velocity control with gravity compensation for magnetic helical microswimmers," Advanced Robotics, vol. 25, no. 8, pp. 1007 - 1028, 2011

[15] M. P. Kummer, J. J. Abbott, S. Dinser, and B. J. Nelson, "Artificial vitreous humor for in vitro experiments," in Proc. of IEEE Int. Conf. Engineering in Medicine and Biology Society, 2007, pp. 6406-6409.

[16] L. A. Negron, F. Viola, E. P. Black, C. A. Toth, and W. F. Walker, "Development and characterization of a vitreous mimicking material for radiation force imaging," IEEE Transactions on Ultrasonics, Ferroelectrics and Frequency Control, vol. 49, no. 11, pp. 1543 -1551, 2002

[17] Z.-J. Chen, G. T. Gillies, W. C. Broaddus, S. S. Prabhu, H. Fillmore, R. M. Mitchell, F. D. Corwin, and P. P. Fatouros, "A realistic brain tissue phantom for intraparenchymal infusion studies," Journal of Neurosurgery, vol. 101, no. 2, pp. 314-322, 2004

[18] K. Zell, J. I. Sperl, M. W. Vogel, R. Niessner, and C. Haisch, "Acoustical properties of selected tissue phantom materials for ultrasound imaging," Physics in Medicine and Biology, vol. 52, no. 20, p. N475, 2007. 